\title{
Label-free fiber nanograting sensor for real-time in situ early monitoring of cellular apoptosis
}

\author{
Danran Li, ${ }^{a, b}$ Nina Wang, ${ }^{c}$ Tianyang Zhang, ${ }^{c}$ Guangxing Wu, ${ }^{a, b}$ Yifeng Xiong, ${ }^{a, b}$ Qianqian Du, ${ }^{d}$ Yunfei Tian, \\ Weiwei Zhao, ${ }^{c}$ Jiandong Ye, ${ }^{d}$ Shulin Gu, ${ }^{d}$ Yanqing Lu, ${ }^{a, b}$ Dechen Jiang, ${ }^{c, *}$ and Fei Xu $\oplus^{a, b, *}$ \\ ${ }^{a}$ Nanjing University, College of Engineering and Applied Sciences, Nanjing, China \\ ${ }^{b}$ Nanjing University, National Laboratory of Solid State Microstructures, Collaborative Innovation Center of Advanced Microstructures, Nanjing, China \\ 'Nanjing University, School of Chemistry and Chemical Engineering, Nanjing, China \\ ${ }^{d}$ Nanjing University, School of Electronic Science and Engineering, Nanjing, China
}

\begin{abstract}
The achievement of functional nanomodules for subcellular label-free measurement has long been pursued in order to fully understand cellular functions. Here, a compact label-free nanosensor based on a fiber taper and zinc oxide nanogratings is designed and applied for the early monitoring of apoptosis in individual living cells. Because of its nanoscale dimensions, mechanical flexibility, and minimal cytotoxicity to cells, the sensing module can be loaded in cells for long term in situ tracking with high sensitivity. A gradual increase in the nuclear refractive index during the apoptosis process is observed, revealing the increase in molecular density and the decrease in cell volume. The strategy used in our study not only contributes to the understanding of internal environmental variations during cellular apoptosis but also provides a new platform for nonfluorescent fiber devices for investigation of cellular events and understanding fundamental cell biochemical engineering.
\end{abstract}

Keywords: fiber nanogratings; label-free sensor; apoptosis monitoring.

Received Oct. 26, 2021; revised manuscript received Nov. 24, 2021; accepted for publication Dec. 14, 2021; published online Jan. 6, 2022.

(C) The Authors. Published by SPIE and CLP under a Creative Commons Attribution 4.0 International License. Distribution or reproduction of this work in whole or in part requires full attribution of the original publication, including its DOI.

[DOI: 10.1117/1.AP.4.1.016001]

\section{Introduction}

The intracellular microenvironment involves vital physiological characteristics of various cellular compartments. The fabrication of nanoprobes for subcellular measurement is important to fully characterize cellular function. Single-cell interrogation at the nanoscale is carried out mainly via optical approaches, such as the use of fluorescent dyes, ${ }^{1-3}$ quantum dots or nanoparticles, ${ }^{4-11}$ nanofiber probes, ${ }^{12,13}$ single nanowire probes, ${ }^{14-19}$ and planar photonic crystal nanowaveguides; ${ }^{20}$ mechanical approaches, such as the use of atomic force microscopy probes $^{21-23}$ and nanobeam arrays $;{ }^{24}$ and electrochemical approaches, such as the utilization of single metal nanowires, ${ }^{25,26}$ pillars, or tube electrodes..$^{27-31}$ Dyes and quantum-dot-based probes have attracted much attention in biology, attributable to their photoluminescence (PL), and achieved great success in measuring the temperature of cells and their organelles, ${ }^{32,33}$ as they can be easily phagocytosed by cells

*Address all correspondence to Fei Xu, feixu@nju.edu.cn; Dechen Jiang, dechenjiang@nju.edu.cn due to their ultrasmall dimensions and are quite robust against external disturbances. However, these techniques suffer from some limitations, such as fluorescence bleaching and background fluorescence interruption. Optical nanofibers have become increasingly popular owing to their excellent optical waveguiding property, high flexibility, and ease of integration ${ }^{34}$ and thus have been applied for the detection of intracellular $\mathrm{pH}^{14}$ and ionic concentrations. ${ }^{15}$ Notably, the existing fiber probes are passive in nature and serve only as conduits to guide light signals into/from a cell but not as a complete optical functional module for physical or chemical label-free sensing. Accordingly, most nanoprobe techniques still have to modify other nanoparticles, resulting in the low functional integration of structures.

The miniaturization of optical modules based on fibers is continuously pursued by researchers. Limited by manufacturing techniques, the scale of all-fiber functional modules is on the order of tens or hundreds of micrometers, ${ }^{35,36}$ which is not applicable compared to the sizes of most common biological cells. Fortunately, one-dimensional nanowires compensate for this deficiency. Their smaller dimensions and high robustness make 
(a)

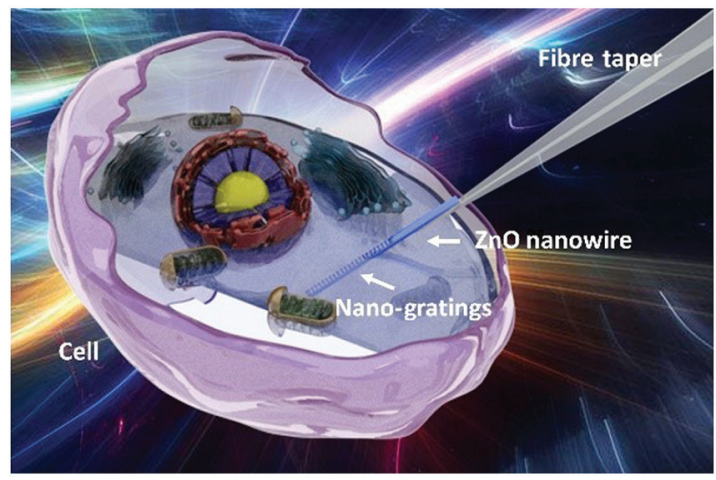

(b)

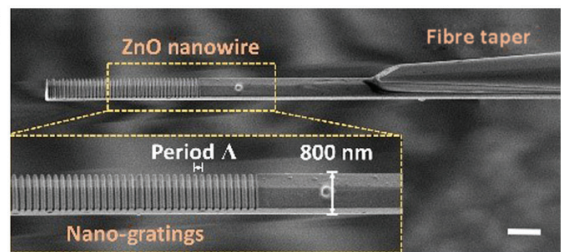

(c)

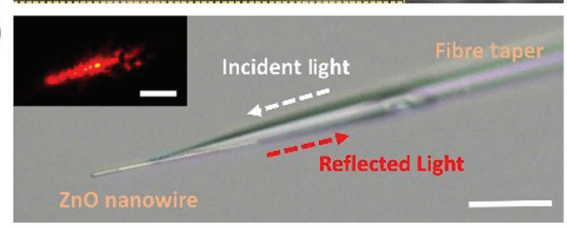

Fig. 1 Schematic illustration and design of the fiber nanogratings sensor. (a) Schematic diagram of the $\mathrm{ZnO}$ nanogratings integrated on a fiber bioprobe. (b) SEM image of the fiber sensor, with a nanowire diameter of $800 \mathrm{~nm}$. Scale bar, $1 \mu \mathrm{m}$. Inset, details of the nanogratings in the yellow dashed rectangle. (c) Microscope image of the fiber sensor with the nanogratings against a bright field. Scale bar, $10 \mu \mathrm{m}$. Inset, microscope image of the nanowire against a dark field, with incident light of $633 \mathrm{~nm}$. Scale bar, $5 \mu \mathrm{m}$.

them suitable for optical resonators, ${ }^{37}$ couplers,${ }^{38}$ gratings,${ }^{39}$ and lasers. ${ }^{40,41}$ However, most of the current optical nanodevices are fixed on substrates, lacking portability and the plug-and-play feature. Moreover, local far-field incident light also implies low-energy efficiency. For an indeed label-free device, the excitation, transmission, detection, and collection of light signals should be integrated in the same device without any chemical modification. Only in this way can the integration of the device, the convenience of manipulation, and the efficiency of sensing be greatly improved. Under these circumstances, the combination of nanowire optical devices and fiber probes provides a novel configuration. Adhered to the tip of a tapered fiber, the nanodevice can be freely moved, can be flexibly directed to anywhere, and can safely penetrate the plasma membrane and enter biological cells, which is potentially useful in single-cell interrogation with high resolution and efficiency.

In this paper, we develop a compact label-free nanosensor based on a complete functional module composed of a fiber taper integrated with zinc oxide $(\mathrm{ZnO})$ nanogratings for the early monitoring of apoptosis in living cells, as illustrated in Fig. 1(a). Cellular apoptosis, which is involved in cell turnover in many healthy adult tissues, is responsible for the focal elimination of cells during normal embryonic development. The visualization and monitoring of early apoptosis and the real-time tracking of cell status with high sensitivity in living cells are highly desired. We choose a single $\mathrm{ZnO}$ nanowire not only because of its low toxicity and high biological compatibility, but also because it can serve as a waveguiding matrix and has low transmission loss and a relatively high refractive index (RI, $n=$ 1.9831 at $655 \mathrm{~nm}$ ), which are required for strong light confinement in the nanowire, especially in aqueous environments $(n=1.3310$ at $655 \mathrm{~nm})$. Moreover, the uniform diameter of the nanowire results in less physical damage to cellular structures and functions than caused by a conical optical fiber probe when penetrating a cell. With optical nanogratings etched on the $\mathrm{ZnO}$ nanowire, the integrated fiber sensor is functionalized with the capability of simultaneously sending and receiving optical signals, being sensitive to changes in the surrounding environment, and can realize rapid, accurate, and real-time sensing by being physically located in a single living cell. In our study, we find that the RI of the nucleus is higher than that of the cytoplasm in HeLa cells. More importantly, a gradual increase in nuclear RI during the apoptosis process after the invasion of hydrogen peroxide is observed, which illustrates an increase in molecular density and a decrease in cell volume. ${ }^{42}$ Overall, the quantitative probing and analysis of the RI naturally present in single living cells during apoptosis may offer insight into the life activities within cells. Additionally, our strategy creates a precedent that allows a passive fiber nanodevice for in situ, dynamic intracellular monitoring and may provide new ideas for a number of novel studies combining photonics with biochemistry.

\section{Results and Discussion}

\subsection{Architectures of the Fiber Nanogratings Sensor}

The nanosensor was fabricated by bonding $\mathrm{ZnO}$ nanogratings on the tip of an optical fiber taper (see Sec. S1 in the Supplementary Material). The manufactured fiber taper had a conical transition region generally several millimeters in length and a long pigtail to allow prompt linking to other optical fiber components. Light traveling along the fiber can be effectively coupled into the nanogratings, where part of the light is reflected back. Predictably, thinner nanowires cause fewer injuries to cell membranes during the puncture process; however, a larger diameter is more desirable in terms of light confinement and mechanical robustness. Therefore, we prioritized nanowires with diameters of 500 to $800 \mathrm{~nm}$. The microscope image [see Fig. 1(c)] of a typical probe shows that a single $\mathrm{ZnO}$ nanowire with gratings in the visible band $(\sim 660 \mathrm{~nm})$ etched on the front end was successfully attached onto the tip of the fiber taper. The scanning electron microscopy (SEM) micrograph of the nanostructured probe [see Fig. 1(b)] shows that the gratings on the $\mathrm{ZnO}$ nanowire included 40 shallow grooves with a period $\Lambda=169 \mathrm{~nm}$, resulting in a total length $<7 \mu \mathrm{m}$, which is two orders of magnitude shorter than gratings fabricated in conventional optical fibers. Each groove was $\sim 100 \mathrm{~nm}$ deep and $\sim 80 \mathrm{~nm}$ wide and located at a position with a local radius of $\sim 400 \mathrm{~nm}$. The configuration has good manufacturing reproducibility and stability.

For a highly efficient nanowire probe, the optical output is closely confined to the $\mathrm{ZnO}$ nanowire, offering highly localized 
illumination. Owing to the excellent waveguiding properties of both the optical fiber and $\mathrm{ZnO}$ nanowire, the optical transmission loss is generally concentrated in the coupling region between them. As illustrated in our previous research, ${ }^{43}$ the normalized optical power flow coupled into the nanowire depends on the propagating constants of the two waveguides, which are related to the diameters of the two waveguides in the coupling region. To improve the coupling efficiency, we designed and fabricated probes with appropriate sizes. Figure 1(b) clearly shows that most light transmitted in the fiber probe is coupled into the nanowire. Benefitting from the high $\mathrm{RI}$ of $\mathrm{ZnO}$, the external environmental RI hardly affects the optical coupling and light propagation.

\subsection{Optical Characteristics and Calibration in vitro}

The setup used to test the nanogratings sensor is illustrated in Sec. S3 in the Supplementary Material. Figure 2(a) shows the typical reflectance spectrum of the fiber probe immersed in deionized water (see Sec. S3 in the Supplementary Material). To investigate the function of the $\mathrm{ZnO}$ nanogratings, we measured the reflectance spectra of the fiber taper without (black curve) and with (blue curve) the $\mathrm{ZnO}$ nanogratings. It is evident that the sole fiber taper reflects little light, while by contrast, the $\mathrm{ZnO}$ nanogratings have a characteristic reflection wavelength at $\sim 658 \mathrm{~nm}$. The ratio of reflectance is $\sim 30 \%$ compared to the end face of a sliced fiber. The extinction ratio, which indicates the ratio of the maximum to minimum reflection intensities, is $\sim 11 \mathrm{~dB}$, and the full-width at half-maximum is $\sim 8 \mathrm{~nm}$.

A distinctive feature of optical gratings is that they are very sensitive to the RI of the external environment. ${ }^{44}$ With the change in RI, we discovered an apparent redshift in the peak reflection wavelength [see Fig. 2(b) and Sec. S5 in the Supplementary Material]. Figure 2(c) (black line) demonstrates the linear relationship between the peak reflection wavelength of the nanosensor and the surrounding RI, with a sensitivity of $\sim 83 \mathrm{~nm} / \mathrm{RIU}$. The experimental result is in good agreement with the theoretical simulation (see Sec. S2 in the Supplementary Material). After calibration, the RI of pure phosphate buffered saline (PBS, HyClone) was measured to be 1.3345 , again similar to previously reported data. ${ }^{45}$

Using the shift in the spectrum is a common method in optical sensing ${ }^{46}$ whereas the increase in temperature resulting from the high light power cannot be neglected, as it may cause predictable thermal damage to the cells. To make the experimental conditions more rigorous, a low-power laser at $655 \mathrm{~nm}$ was used as the light source to conduct single-cell sensing. Figure 2(c) (red line) shows the reflection power of $655 \mathrm{~nm}$ derived from Fig. 2(b) (red dashed line) with increasing RI. The power sensitivity is $\sim 1260 \% / \mathrm{RIU}$. The linear relationship (a)

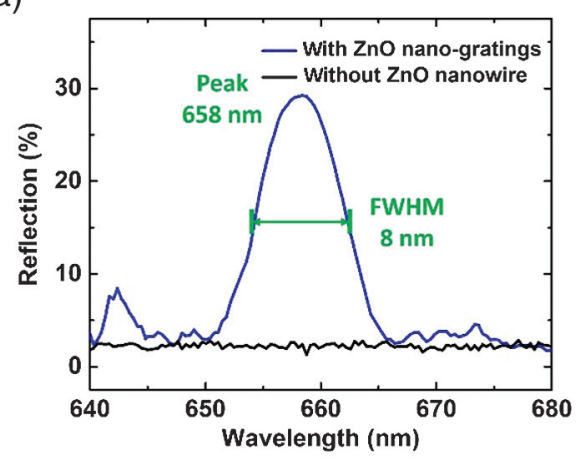

(c)

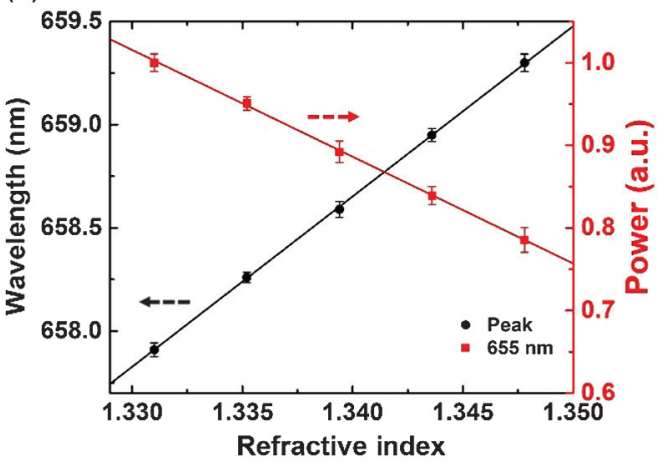

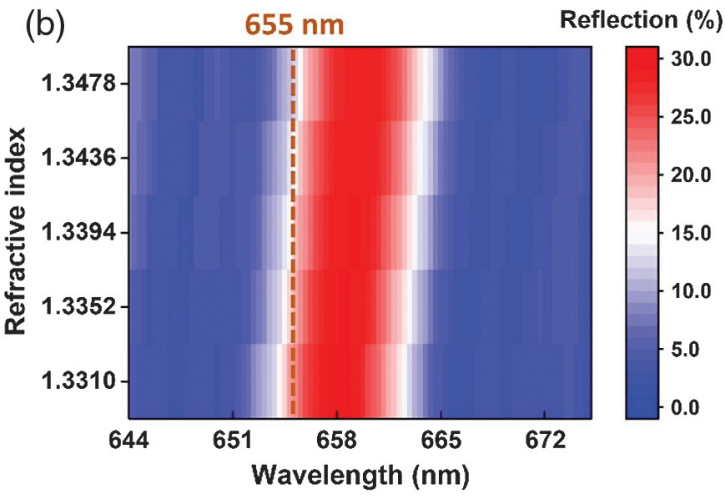

(d)

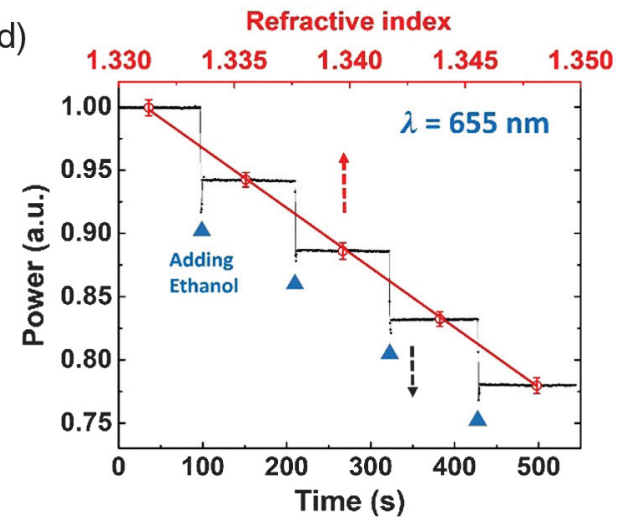

Fig. 2 Optical characteristics and RI sensitivity of the nanosensor. (a) Reflectance spectra of the fiber probe with (blue line) and without (black line) $\mathrm{ZnO}$ nanogratings. The peak wavelength is $658 \mathrm{~nm}$. (b) Color map of spectra with different external Rls. (c) Shift in the peak reflection wavelength (black line) and the change in power at $655 \mathrm{~nm}$ (red line) as a function of the external RI. The red line is derived from the values in (b). (d) Dynamic response of the reflection power at $655 \mathrm{~nm}$ with the addition of ethanol (black line) and the power at $655 \mathrm{~nm}$ as a function of the external RI (red line). The figure closely coincides with the red line in (c). 
indicates that measuring the power change of a single wavelength is another feasible optical sensing approach. As illustrated in Fig. 3(a), the laser $(\sim 800 \mathrm{nW})$ was injected into the fiber nanosensor and the reflected signal was collected with a power meter (see Sec. S4 in the Supplementary Material). The optical power passing through the fiber taper was hundreds of nanowatts, but the light was not completely incident into the cell because nearly half the light was lost in the coupling region between the fiber taper and the nanowire. The power threshold before cell damaging and death involves the exposure time, the size of the cell, and the volume of the solution, ${ }^{47}$ but the power we used is far lower than the threshold. Such low optical power can have little impact on cell activities, as heat dissipates quickly in PBS solution. The calibration indicates that with increasing RI, the reflection power at $655 \mathrm{~nm}$ will gradually decrease within a certain range [see Fig. 2(d), black line]. Therefore, the RI information can also be obtained by measuring the light power at a single wavelength, with the experimental sensitivity of the nanosensor being $\sim 1280 \% /$ RIU for an RI ranging from 1.330 to 1.350 (red line). This result is generally consistent with the experimental results presented in Fig. 2(c) (red line).

\subsection{Safe Penetration Tests}

To demonstrate that the nanowire probes incurred negligible cell membrane damage during insertion and extraction, fluorescent dyes were used to verify whether the cell membranes were ruptured (see Sec. S8 in the Supplementary Material). Optical fiber probes with and without a $\mathrm{ZnO}$ nanowire were (a)

(b)

(c)

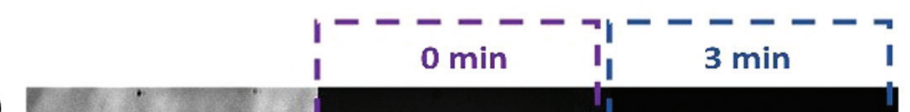

(d)

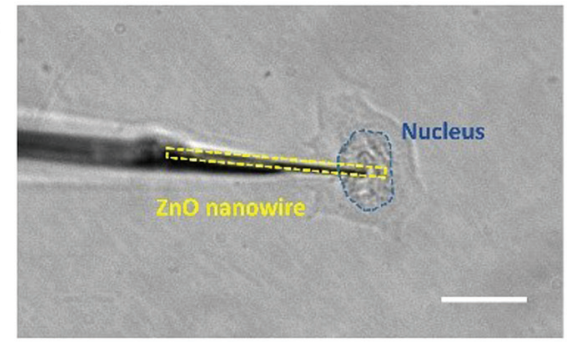

(e)
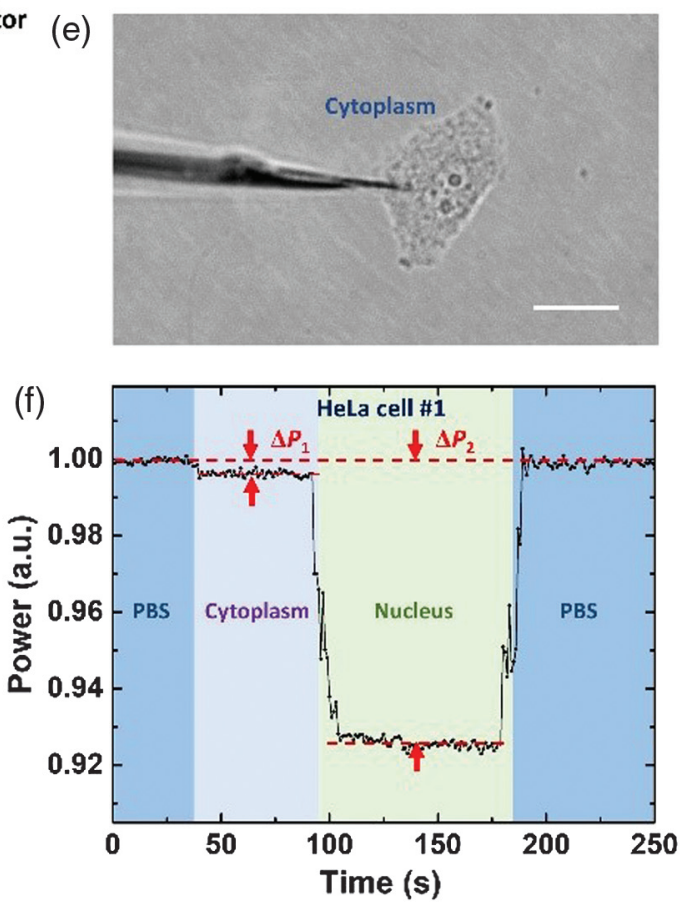

Fig. 3 Intracellular calibration and measurements of the nanosensor. (a) Schematic diagram of the experimental setup used to calibrate the RI sensitivity of the probe and measure the intracellular RI of HeLa cells. A low-power laser at $655 \mathrm{~nm}(\sim 800 \mathrm{nW})$ was injected into the pigtail of the fiber probe through port 2 of a visible circulator. The output light reflected at the nanogratings was transmitted through port 3 of the circulator, which was connected to an optical power meter to record the reflection power. Microscope and PL images of the tested cell (in red circle) and other reference cells. The tested cell was penetrated by a fiber probe with an $\sim 500 \mathrm{~nm}$ conical tip (b) and a $\mathrm{ZnO}$ nanograting-integrated fiber probe with an $~ 800 \mathrm{~nm}$ tip (c). Scale bar, $40 \mu \mathrm{m}$. Microscope images of the $\mathrm{ZnO}$ nanowire-integrated fiber probe inserted into the (d) nucleus and (e) cytoplasm of single HeLa cells. Scale bar, $10 \mu \mathrm{m}$. (f) Normalized reflection power in the cytoplasm and nucleus of a single HeLa cell, indicating the difference in RI. 
manipulated to penetrate HeLa cells. HeLa was the first human cell line established in culture and has since become the most widely used human cell line in biological research. ${ }^{48}$ Figure 3(b) shows the tested cell (red circle) after insertion of a fiber probe with an $\sim 500 \mathrm{~nm}$ tip. But for the conical structure, the actual damaged area of the membrane was larger than the tip diameter. The PL intensity decreased significantly compared with that of other cells $3 \mathrm{~min}$ after puncture, which indicated that the cell membrane was likely to be damaged, resulting in the outflow of dyes. Figure 3(c) shows the tested cell (red circle) after insertion of the $\mathrm{ZnO}$ nanowire-integrated fiber probe with an $\sim 800 \mathrm{~nm}$ tip. Owing to the uniform diameter of the nanowire, the PL intensity of the tested cell showed no apparent differences compared with the surrounding cells within 3 min after puncture, indicating that the cell membrane was still relatively intact. The observation of fluorescence from the cells shows that the cells continued to hydrolyse fluorescein diacetate to generate fluorescein inside the cells. Therefore, it is feasible to use the $\mathrm{ZnO}$ nanowire-integrated fiber sensor to implement intracellular measurements.

\subsection{Intracellular Experiments on RI Sensing}

Figures 3(d) and 3(e) show that the nucleus and cytoplasm regions of single HeLa cells are clearly visible in the microscope images even after insertion of the $\mathrm{ZnO}$ nanograting-integrated fiber sensor (see Sec. S7 in the Supplementary Material). The nanowire is robust against cell penetration, with no obvious mechanical deformation. As illustrated in Fig. 3(f), the normalized reflection power declined by $\sim 0.5 \%$ when the probe was transferred from PBS into the cytoplasm and decreased by $\sim 7 \%$ when the probe entered the nucleus. The tiny fluctuations $(\sim 0.08 \%)$ of real-time power stemmed from the variations of the light source power and the unevenness of intracellular microenvironments. Notably, the experiment was carried out in a constant temperature environment to eliminate the influence of temperature fluctuations. The probe did not stay in the cytoplasm after measuring the RI of the nucleus, so the information from nucleus-cytoplasm-PBS was not apparent. If we extract the probe out of the cell slower, the information will be basically similar to the former. We tested four single HeLa cells, and the probe was cleaned with PBS solution before and after each test to avoid contamination. The experimental results in Table 1 and Sec. S9 in the Supplementary Material indicate that the mean RI of the cytoplasm and the nucleus in the HeLa cells was 1.3348 and 1.3402 , respectively. The distribution of substances in the cells is possibly nonuniform, and it can be inferred that the RI results of the single HeLa cells relate to the position of the probe. Since the RI is generally proportional to the

Table $1 \mathrm{RI}$ measured in the cytoplasm and nucleus of four HeLa cells.

\begin{tabular}{lcccc}
$\begin{array}{c}\text { Cell } \\
\text { No. }\end{array}$ & $\begin{array}{c}\text { Decrease in } \\
\text { power }\left(\Delta P_{1}\right) \text { in } \\
\text { cytoplasm }(\%)\end{array}$ & $\begin{array}{c}\mathrm{RI} \text { in } \\
\text { cytoplasm }\end{array}$ & $\begin{array}{c}\text { Decrease in } \\
\text { power }\left(\Delta P_{2}\right) \text { in } \\
\text { nucleus }(\%)\end{array}$ & $\begin{array}{c}\mathrm{RI} \text { in } \\
\text { nucleus }\end{array}$ \\
\hline 1 & 0.34 & 1.3348 & 7.41 & 1.3403 \\
2 & 0.69 & 1.3350 & 9.59 & 1.3420 \\
3 & 0.13 & 1.3346 & 5.62 & 1.3389 \\
4 & 0.25 & 1.3347 & 6.39 & 1.3395 \\
\hline
\end{tabular}

molecular concentration, which is related to the viscosity of solutions, the molecular density of the cytoplasm is deduced to be slightly higher than that of PBS and the molecular density of the nucleus is much higher than that of the cytoplasm. The relatively stable fluctuation of the reflection power also indicated that the signal light had little thermal effect on the cells. Previous studies using optical diffraction tomography also drew similar conclusions. ${ }^{42}$ Compared with traditional far-field detection techniques, our method is more energy efficient, as only very low-power probe light is required.

\subsection{Intracellular Dynamic Monitoring of Cellular Apoptosis}

Cell apoptosis is an integral part of normal development and the maturation cycle. Despite the importance of this process, the mechanisms underlying cell death are still poorly understood. Previous work has shown the RI changes of cells can indirectly reflect different intracellular behaviors. ${ }^{42}$ Therefore, we hope to discover the connection between nuclear RI and cell apoptosis under external stimuli, which is helpful for further understanding of cell life events and development of diseases. The advantage of using nanogratings instead of fluorescence for single-cell detection is that the nanoconfiguration is more stable and reliable for long-term monitoring because fluorescence suffers from quenching and bleaching. After the probe punctured the nucleus of a HeLa cell, $10 \mu \mathrm{L}$ of $20 \mathrm{mmol} / \mathrm{L} \mathrm{H}_{2} \mathrm{O}_{2}$ PBS solution was injected into the medium. Hydrogen peroxide $(n=1.3350$ at $655 \mathrm{~nm}$ ) is a strong oxidant that breaks down cell membranes and is thus fatal to cells. The microscope images of the detected HeLa cells [see Fig. 4(a)-4(d)] show the variation in cell appearance. Simultaneously, a reduction in the reflection intensities was observed in the nucleus, indicating a continuous increase in RI with time [see Fig. 4(e)]. The gradual increase in nuclear RI went through three periods. During the first period, which lasted $\sim 20 \mathrm{~min}$, the process of apoptosis was slow. The cytoplasm was gradually dissolved under the corrosion of hydrogen peroxide, but the morphology of the nucleus did not have a notable impact [see Fig. 4(b)], and the RI of the nucleus increased slowly. During the second period, which lasted $\sim 10 \mathrm{~min}$, large areas of the cytoplasm dissolved, and the nucleus began to shrink [see Fig. 4(c)]. The nuclear RI rapidly increased by $\sim 0.0040$ with decreasing nuclear volume. During the last period, the cells had little vitality, and the nuclear RI tended to become stable. We tested three single HeLa cells, and the mean nuclear RI after cell apoptosis was 1.3442, as indicated in Table 2 and Sec. S12 in the Supplementary Material. Although the exact reasons for the changes in the RI of the nucleus are still unknown, our results imply that the shape and volume of the cell are at least partly responsible. The numbers of pixels that the cell nucleus occupied in the microscope images [see Figs. 4(a)-4(d)] during the process of cell apoptosis were counted, which could roughly reflect the volume of the cell nucleus [see Fig. 4(e), blue circles]. The volume of the cell nucleus gradually contracted, leading to an increase in molecular density, which is one of the possible reasons for the increase in the nuclear RI.

A mature fluorescence technique was used to examine the cell viability of the tested cells and other normal cells during the process of apoptosis (see Secs. S10 and S11 in the Supplementary Material). The fluorescent dye we chose presents little toxicity to cells, can infiltrate through cell membranes, and is often used for nuclear staining and cell apoptosis 


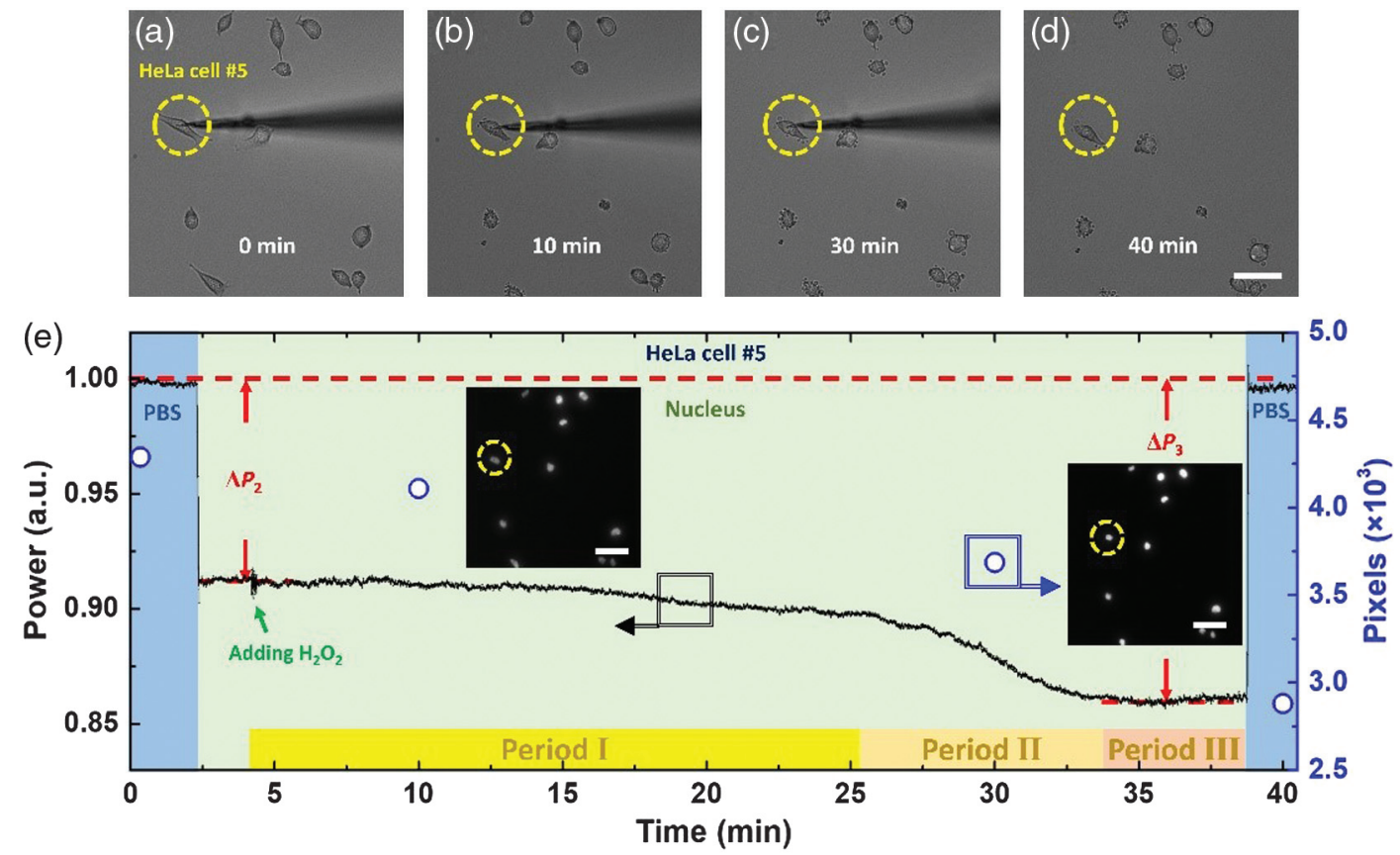

Fig. 4 Real-time long-term early monitoring of HeLa cell apoptosis. Microscope images of single tested HeLa cells (yellow dashed circle) and other reference cells during the apoptosis process. (a) Immediately after the addition of $\mathrm{H}_{2} \mathrm{O}_{2}$, (b) 10 min after the addition of $\mathrm{H}_{2} \mathrm{O}_{2}$, (c) 30 min after the addition of $\mathrm{H}_{2} \mathrm{O}_{2}$, and (d) apoptotic cells 40 min after the addition of $\mathrm{H}_{2} \mathrm{O}_{2}$, scale bar, $20 \mu \mathrm{m}$. (e) Normalized reflection power in the nucleus of a single tested HeLa cell during apoptosis under the stimulation of $\mathrm{H}_{2} \mathrm{O}_{2}$ (black line), indicating a continuous increase in the nuclear RI. The numbers of pixels that the cell nucleus occupies in the microscope images (blue circle) roughly indicate the volume of the nucleus. Inset, PL images of living and apoptotic HeLa cells. Scale bar, $20 \mu \mathrm{m}$.

Table 2 Measured RI of the nucleus in three apoptotic HeLa cells.

\begin{tabular}{|c|c|c|c|c|}
\hline $\begin{array}{l}\text { Cell } \\
\text { No. }\end{array}$ & $\begin{array}{c}\text { Decrease in } \\
\text { power }\left(\Delta P_{2}\right) \text { in } \\
\text { nucleus before } \\
\text { apoptosis }(\%)\end{array}$ & $\begin{array}{l}\text { Nuclear } \\
\text { RI before } \\
\text { apoptosis }\end{array}$ & $\begin{array}{l}\text { Decrease in } \\
\text { power }\left(\Delta P_{3}\right) \text { in } \\
\text { nucleus after } \\
\text { apoptosis }(\%)\end{array}$ & $\begin{array}{l}\text { Nuclear } \\
\text { RI after } \\
\text { apoptosis }\end{array}$ \\
\hline 5 & 8.16 & 1.3408 & 13.89 & 1.3453 \\
\hline 6 & 6.92 & 1.3399 & 11.53 & 1.3435 \\
\hline 7 & 7.59 & 1.3405 & 11.92 & 1.3438 \\
\hline
\end{tabular}

tests (see Sec. S10 in the Supplementary Material). ${ }^{49}$ As shown in Fig. 4(e), the intensity of the PL signal underwent an apparent increase 40 min after $\mathrm{H}_{2} \mathrm{O}_{2}$ was injected, confirming that the cells had indeed been apoptotic. Moreover, the PL intensity of the tested cell (yellow dashed circle) had no obvious difference from that of other surrounding cells, which again indicated that the $\mathrm{ZnO}$ nanogratings had little influence on the cell life states.

\section{Conclusion}

We developed a versatile, biocompatible, portable, and reusable $\mathrm{ZnO}$ nanograting-integrated label-free nanosensor that enables in situ early monitoring of cellular apoptosis. The nanodevice integrates signal transmission, sensing, and collection, with high integration, operability, and sensitivity. By inserting the nanoscale functional optical module into single living HeLa cells, we can detect different features between the nucleus and cytoplasm. The highly flexible and robust nanosensor allowed us to reveal that the mean RI of the nucleus is 0.0054 higher than that of the cytoplasm in single HeLa cells, which to some extent verifies the higher molecular density of the nucleus than that of cytoplasm. Moreover, the stable and nondegradable configuration is promising for the long-term monitoring of cellular environmental variations, which are universally present in all cell life activities. We observed a decrease in the nuclear volume and detected the nuclear RI during cell apoptosis. The results show that the final mean RI is 0.0040 higher than that of normal living cells, linking the RI and the volume of the nucleus to the state of living cells. We consider that it is feasible to detect and compare the differences between normal living cells and cancer cells using the same sensing device. The application of the nanoprobe is only limited by the size of the cell and has no requirement for the type of cells. This study is the first to realize apoptosis sensing and tracking with nanogratings in living cells. Real-time probing of cellular apoptosis will not only attract more attention to cell life events but also broaden the application scope of fiber nanosensors.

\section{Acknowledgments}

This research was sponsored by the National Natural Science Foundation of China (Nos. 61925502 and 62135007). The authors have no conflicts of interest to declare. 


\section{References}

1. A. Colom et al., "A fluorescent membrane tension probe," Nat. Chem. 10(11), 1118-1125 (2018).

2. J. S. Donner et al., "Mapping intracellular temperature using green fluorescent protein," Nano Lett. 12(4), 2107-2111 (2012).

3. K. Okabe et al., "Intracellular temperature mapping with a fluorescent polymeric thermometer and fluorescence lifetime imaging microscopy," Nat. Commun. 3(1), 705 (2012).

4. J. M. Yang, H. Yang, and L. Lin, "Quantum dot nanothermometers reveal heterogeneous local thermogenesis in living cells," ACS Nano 5(6), 5067-5071 (2011).

5. X. Chen et al., "Imaging the transient heat generation of individual nanostructures with a mechanoresponsive polymer," Nat. Commun. 8(1), 1498 (2017).

6. G. Kucsko et al., "Nanometre-scale thermometry in a living cell," Nature 500(7460), 54-58 (2013).

7. Z. Chen et al., "Single gold@ silver nanoprobes for real-time tracing the entire autophagy process at single-cell level," J. Am. Chem. Soc. 137(5), 1903-1908 (2015).

8. O. A. Savchuk et al., "Thermochromic upconversion nanoparticles for visual temperature sensors with high thermal, spatial and temporal resolution," J. Mater. Chem. C 4(27), 6602-6613 (2016).

9. X. Zhu et al., "Temperature-feedback upconversion nanocomposite for accurate photothermal therapy at facile temperature," Nat. Commun. 7(1), 10437 (2016).

10. P. C. Wuytens et al., "Gold nanodome-patterned microchips for intracellular surface-enhanced Raman spectroscopy," Analyst 140(24), 8080-8087 (2015).

11. K. Yum et al., "Mechanochemical delivery and dynamic tracking of fluorescent quantum dots in the cytoplasm and nucleus of living cells," Nano Lett. 9(5), 2193-2198 (2009).

12. T. Vo-Dinh et al., "Antibody-based nanoprobe for measurement of a fluorescent analyte in a single cell," Nat. Biotechnol. 18(7), 764-767 (2000).

13. Q. Yang et al., "Fiber-optic-based micro-probe using hexagonal 1-in-6 fiber configuration for intracellular single-cell $\mathrm{pH}$ measurement," Anal. Chem. 87(14), 7171-7179 (2015).

14. R. Yan et al., "Nanowire-based single-cell endoscopy," Nat. Nanotechnol. 7(3), 191-196 (2012).

15. J. Lee et al., "Quantitative probing of $\mathrm{Cu}^{2+}$ ions naturally present in single living cells," Adv. Mater. 28(21), 4071-4076 (2016).

16. J. H. Lee et al., "Spontaneous internalization of cell penetrating peptide-modified nanowires into primary neurons," Nano Lett. 16(2), 1509-1513 (2016).

17. X. Cao et al., "Single silicon nanowire-based fluorescent sensor for endogenous hypochlorite in an individual cell," Adv. Biosyst. 2(12), 1800213 (2018).

18. C. Chiappini et al., "Biodegradable silicon nanoneedles delivering nucleic acids intracellularly induce localized in vivo neovascularization," Nat. Mater. 14(5), 532-539 (2015).

19. W. Hong et al., "Nanoscale label-free bioprobes to detect intracellular proteins in single living cells," Sci. Rep. 4(1), 6179 (2014).

20. G. Shambat et al., "Single-cell photonic nanocavity probes," Nano Lett. 13(11), 4999-5005 (2013).

21. A. Meister et al., "FluidFM: combining atomic force microscopy and nanofluidics in a universal liquid delivery system for single cell applications and beyond," Nano Lett. 9(6), 2501-2507 (2009).

22. P. Knittel et al., "Focused ion beam-assisted fabrication of soft high-aspect ratio silicon nanowire atomic force microscopy probes," Ultramicroscopy 179, 24-32 (2017).

23. O. Guillaume-Gentil et al., "Tunable single-cell extraction for molecular analyses," Cell 166(2), 506-516 (2016).

24. F. Santoro et al., "Revealing the cell-material interface with nanometer resolution by focused ion beam/scanning electron microscopy," ACS Nano 11(8), 8320-8328 (2017).
25. S. M. Yoo et al., "Electrotriggered, spatioselective, quantitative gene delivery into a single cell nucleus by Au nanowire nanoinjector," Nano Lett. 13(6), 2431-2435 (2013).

26. G. Lu et al., "Live-cell SERS endoscopy using plasmonic nanowire waveguides," Adv. Mater. 26(30), 5124-5128 (2014).

27. P. D. Tovee et al., "Nanoscale resolution scanning thermal microscopy using carbon nanotube tipped thermal probes," Phys. Chem. Chem. Phys. 16(3), 1174-1181 (2014).

28. R. Pan et al., "Resistive-pulse sensing inside single living cells," J. Am. Chem. Soc. 142(12), 5778-5784 (2020).

29. H. Liu et al., "A multiparameter $\mathrm{pH}$-sensitive nanodevice based on plasmonic nanopores," Adv. Funct. Mater. 28(1), 1703847 (2018).

30. Y. Zhang et al., "Spearhead nanometric field-effect transistor sensors for single-cell analysis," ACS Nano 10(3), 3214-3221 (2016).

31. R. Singhal et al., "Multifunctional carbon-nanotube cellular endoscopes," Nat. Nanotechnol. 6(1), 57-64 (2011).

32. D. Chrétien et al., "Mitochondria are physiologically maintained at close to 50 ${ }^{\circ} \mathrm{C}, "$ PLoS Biol. 16(1), e2003992 (2018).

33. S. Arai et al., "Mitochondria-targeted fluorescent thermometer monitors intracellular temperature gradient," Chem. Commun. 51(38), 8044-8047 (2015).

34. X. Fan et al., "Sensitive optical biosensors for unlabeled targets: a review," Anal. Chim. Acta 620(1-2), 8-26 (2008).

35. J. L. Kou et al., "Demonstration of a compact temperature sensor based on first-order Bragg grating in a tapered fiber probe," Opt. Express 19(19), 18452-18457 (2011).

36. J. L. Kou et al., "Miniaturized fiber taper reflective interferometer for high temperature measurement," Opt. Express 18(13), 1424514250 (2010).

37. Z. Hu, X. Guo, and L. Tong, "Freestanding nanowire ring laser," Appl. Phys. Lett. 103(18), 183104 (2013).

38. X. Xing et al., "Ultracompact photonic coupling splitters twisted by PTT nanowires," Nano Lett. 8(9), 2839-2843 (2008).

39. A. Fu et al., "Widely tunable distributed Bragg reflectors integrated into nanowire waveguides," Nano Lett. 15(10), 6909-6913 (2015).

40. R. Röder et al., "Ultrafast dynamics of lasing semiconductor nanowires," Nano Lett. 15(7), 4637-4643 (2015).

41. J. Tatebayashi et al., "Room-temperature lasing in a single nanowire with quantum dots," Nat. Photonics 9(8), 501-505 (2015).

42. T. K. Kim et al., "Physicochemical properties of nucleoli in live cells analyzed by label-free optical diffraction tomography," Cells 8(7), 699 (2019).

43. D. R. Li et al., "Ethanol gas sensor based on a hybrid polymethyl methacrylate-silica microfiber coupler," J. Lightwave Technol. 36(10), 2031-2036 (2018).

44. F. Chiavaioli et al., "Biosensing with optical fiber gratings," Nanophotonics 6(4), 663-679 (2017).

45. S. Cohen-Maslaton et al., "Cell and nucleus refractive-index mapping by interferometric phase microscopy and rapid confocal fluorescence microscopy," J. Biophotonics 13(9), e202000117 (2020).

46. F. Chiavaioli, "Recent development of resonance-based optical sensors and biosensors," Optics 1(3), 255-258 (2020).

47. A. Calabuig et al., "Investigating fibroblast cells under 'safe' and 'injurious' blue-light exposure by holographic microscopy," $J$. Biophotonics 10(6-7), 919-927 (2017).

48. J. J. M. Landry et al., "The genomic and transcriptomic landscape of a HeLa cell line" G3-Genes Genom. Genet. 3(8), 1213-1224 (2013).

49. C. Huang et al., "Long-term blue light exposure induces RGC-5 cell death in vitro: involvement of mitochondria-dependent apoptosis, oxidative stress, and MAPK signaling pathways," Apoptosis 19(6), 922-932 (2014).

50. T. Erdogan, "Fiber grating spectra," J. Lightwave Technol. 15(8), 1277-1294 (1997).

Biographies of the authors are not available. 